\title{
Clinicopathologic Features of Primary Merkel Cell Carcinoma: A Detailed Descriptive Analysis of a Large Contemporary Cohort
}

Jennifer L. Schwartz, MD, ${ }^{*}$ Christopher K. Bichakjian, MD, ${ }^{*}$ Lori Lowe, MD, ${ }^{* \dagger}$ Kent A. Griffith, MPH, MS, ${ }^{*}$ Marcus L. Frohm, MD, ${ }^{*}$ Douglas R. Fullen, MD,${ }^{\dagger \dagger}$ James A. Hayman, MD, ${ }^{\S}$ Christopher D. Lao, MD, MPH, ${ }^{\Uparrow}$ Khooshbu S. Shah, MD, ${ }^{\circledR}$ Scott A. McLean, MD, PhD, ** Carol R. Bradford, MD, ${ }^{*}$ Timothy M. Johnson, MD, ${ }^{* * *^{\dagger \dagger}}$ and Sandra L. Wong, MD ${ }^{\dagger \dagger}$

BACKGROUND Little uniformity exists in the clinical and histologic variables reported with primary Merkel cell carcinoma (MCC).

OBJECTIVE To provide a rigorous descriptive analysis of a contemporary cohort and promote the prospective collection of detailed data on MCC for future outcome studies.

METHODS AND MATERIALS A detailed descriptive analysis was performed for clinical and histologic features of 147 patients with 150 primary MCC tumors in a prospectively collected database from 2006 to 2010.

RESULTS The majority (73.5\%) of patients were at American Joint Committee on Cancer clinical stage I or II at presentation, $20.4 \%$ at stage III, and $6.1 \%$ at stage IV. Detailed descriptive clinical and histologic findings are presented.

CONCLUSION Clinical and histologic profiling of primary MCC in the literature is variable and limited. Systematic prospective collection of MCC data is needed for future outcome studies and the ability to compare and share data from multiple sources for this relatively rare tumor.

The authors have indicated no significant interest with commercial supporters.

$\mathrm{M}$ erkel cell carcinoma (MCC) is a potentially aggressive malignancy of the skin. With overall 2-year disease-specific mortality estimated at $28 \%$, MCC has a poorer prognosis than melanoma. ${ }^{1}$ Although rare, the incidence of MCC has tripled in the past 2 decades and continues to increase, generating greater attention for this malignancy. ${ }^{2}$

Various clinical, histologic, and immunohistochemical features have been considered as prognostic indicators. In addition to clinical tumor diameter and presence of metastases, other factors are emerging that may be important in predicting prognosis. Results have been mixed; although recent studies report a positive association between tumor thickness, lymphovascular invasion, and infiltrative histologic growth pattern and poor outcome, ${ }^{3-5}$ there is little uniformity in the clinical and histologic parameters reported with a diagnosis of a primary MCC, making it difficult to compare studies and

\footnotetext{
*Department of Dermatology, University of Michigan Health System, Ann Arbor, Michigan; ${ }^{\dagger}$ Department of Pathology, University of Michigan Health System, Ann Arbor, Michigan; ${ }^{\ddagger}$ Comprehensive Cancer Center Biostatistics Core, University of Michigan Health System, Ann Arbor, Michigan; ${ }^{\S}$ Department of Radiation Oncology, University of Michigan Health System, Ann Arbor, Michigan; "Department of Internal Medicine, University of Michigan Health System, Ann Arbor, Michigan; *Department of Otolaryngology, University of Michigan Health System, Ann Arbor, Michigan; ${ }^{\dagger}$ Department of Surgery, University of Michigan Health System, Ann Arbor, Michigan
} 
examine potential prognostic variables. Furthermore, small sample sizes, attributable to this uncommon diagnosis, negatively affect the power of these studies and support the need for the collection of uniform data that may be shared across institutions.

Our purpose was to report a detailed descriptive analysis of prospectively collected clinical and histologic features in a contemporary cohort. Standardization of MCC data collected also provides an optimal framework for future outcome studies and the comparison and sharing of data.

\section{Methods}

\section{Patients}

The Institutional Review Board at the University of Michigan approved this study. Our prospective MCC database was queried for patients diagnosed with a primary MCC who underwent consultation in the Multidisciplinary MCC Program at the University of Michigan between February 2006 and March 2010. This identified 147 patients with a diagnosis of a new primary MCC. One patient developed a second primary MCC in the study period, and two had had a previous diagnosis of primary MCC in 2000 and 2001. Histopathology was reviewed for diagnostic confirmation by a dermatopathologist at the University of Michigan. A profile, including the histopathologic features below, was reported for each primary MCC. ${ }^{6}$

\section{Variables}

Clinical variables included patient sex, age, race, presence or absence of immunosuppression (medication- or disease-induced immunosuppression), history of other skin cancer (yes/no), history of other non-skin cancer (yes/no), site of the primary MCC (head or neck, trunk, arm, hand, leg or buttock, or foot), and clinical size $(<1,1-2,>2 \mathrm{~cm})$. If the tumor was present at consultation, the faculty physician measured it. Otherwise, the size from the referring physician was recorded, or when not available, size was estimated using patient description, biopsy scar, or gross pathology description. Patients were staged at presentation according to the American Joint Committee on Cancer (AJCC) Seventh Edition (stage I, cutaneous disease only, $\leq 2 \mathrm{~cm}$ maximum tumor dimension; stage II, cutaneous disease only, $>2 \mathrm{~cm}$ maximum tumor dimension; stage III, regional lymph node or in transit disease; stage IV, distant disease). ${ }^{7}$ Clinical size was used for maximum tumor dimension.

Histopathologic variables included greatest histologic horizontal dimension within a transversely serially sectioned specimen (measured in millimeter), tumor thickness (measured in $\mathrm{mm}$ from the granular layer of the epidermis to the deepest extent of tumor invasion; Breslow depth), anatomic level of invasion (Clark level), number of mitoses per square millimeter, tumor growth pattern (circumscribed or infiltrative), and presence or absence of ulceration and angiolymphatic invasion. Mitotic rate was determined by counting the number of dermal mitoses in $1 \mathrm{~mm}^{2}$, starting in the field with the most mitoses. Mitotic rate was not included in the profile during initial accrual stages but was subsequently added to the profile. Tumors with a circumscribed growth pattern demonstrated well-circumscribed tumor nodules with pushing borders. An infiltrative growth pattern was characterized by strands, cords, trabeculae, and single cells of tumor infiltrating dermal collagen or soft tissue. Tumors displaying both patterns were classified as infiltrative.

\section{Results}

One hundred forty-seven patients were identified. Three had two primary tumors, for a total of 150 primary MCCs in this cohort. Seventy women $(47.6 \%)$ and $77(52.4 \%)$ men were identified.

Patient characteristics are reported in Table 1 (age, race, immunosuppression status, history of nonMCC skin cancer and non-skin cancer, and clinical and pathologic stage at presentation). Sentinel lymph node biopsy was performed for pathologic 
TABLE 1. Characteristics of 147 Patients With

Primary Merkel Cell Carcinoma (MCC)

\begin{tabular}{lc} 
Characteristic & Value \\
\hline Age, mean (range) & $70.3(38-91)$ \\
Race, $n$ (\%) & \\
Caucasian & $145(98.6)$ \\
African American & $2(1.4)$ \\
Immunosuppressed, $n(\%)$ & \\
Yes & $14(9.5)$ \\
No & $133(90.5)$ \\
History of non-MCC skin cancer, $n(\%)$ & \\
Yes & $64(43.5)$ \\
No & $83(56.5)$ \\
History of non-skin cancer, $n(\%)$ & \\
Yes & $28(19.0)$ \\
No & $119(81.0)$ \\
Clinical stage at presentation, $n(\%)$ & \\
I & $80(54.4)$ \\
II & $28(19.0)$ \\
III & $30(20.4)$ \\
IV & $9(6.1)$ \\
Pathologic stage at presentation, $n(\%)$ & \\
I & $55(37.4)$ \\
II & $12(8.2)$ \\
III & $71(48.3)$ \\
IV & $9(6.1)$ \\
\hline
\end{tabular}

staging in 98 of $108(90.7 \%)$ patients presenting with localized skin MCC, which upstaged 41 $(38.0 \%)$ patients.

Fourteen $(9.5 \%)$ patients were immunosuppressed: six with renal transplants, one with a lung transplant, four with chronic lymphocyte leukemia (CLL), and three taking immunosuppressive medication for other reasons (idiopathic pulmonary fibrosis, ulcerative colitis, rheumatoid arthritis). All immunosuppressed patients were Caucasian ( 8 male, 6 female). The mean age of presentation in these patients was 59.6, compared with 71.4 years in immunocompetent patients. Eightyone $(55.1 \%)$ patients reported a history of other cancer types, skin and non-skin, at the time of presentation (Table 2).

Main tumor characteristics, including histopathology features, are reported in Table 3. Some characteristics had a smaller total number than the 150
TABLE 2. Summary of Other Cancer Types in

Patients With Merkel Cell Carcinoma

\begin{tabular}{ll} 
Cancer Type & $\mathrm{n}(\%)$ \\
\hline Skin & \\
Basal cell carcinoma & $48(32.7)$ \\
Squamous cell carcinoma including & $31(21.1)$ \\
$\quad$ in situ & \\
Melanoma including in situ & $8(5.4)$ \\
Non-skin & \\
Lymphoma & $6(4.1)$ \\
Prostate & $5(3.4)$ \\
Breast & $5(3.4)$ \\
Chronic lymphocytic leukemia & $4(2.7)$ \\
Lung & $4(2.7)$ \\
Renal cell carcinoma & $2(1.4)$ \\
Thyroid & $1(0.7)$ \\
Bladder & $1(0.7)$ \\
Fallopian tube & $1(0.7)$ \\
\hline
\end{tabular}

total tumors because of nonstandardized histopathology in a minority of cases early in the study time period.

In 64 of 148 primary lesions (43.2\%), clinical size was obtained by measuring the lesion at consultation at the University of Michigan, 28 (18.9\%) were determined from the referring physician's description, $48(32.4 \%)$ were estimated based on scar or patient description, $7(4.7 \%)$ were obtained according to gross pathology description, and the source of clinical size measurement was unknown for one lesion. Lesions of $<1 \mathrm{~cm}$ were more likely to be on the head and neck $(64.9 \%, 37 / 57)$ than in other locations, but lesions $1 \mathrm{~cm}$ or more in clinical diameter were more common in other locations $(67 \%, 61 / 91)$ than on the head and neck.

\section{Discussion}

Although the incidence of MCC is lower than with other cutaneous malignancies, the increasing incidence and potentially aggressive nature have directed attention toward this cancer. In this study, patient and tumor features were examined for the purpose of a detailed descriptive analysis from a contemporary, prospectively collected, singleinstitution database. 
TABLE 3. Tumor Characteristics of 150 Primary Merkel Cell Carcinomas

\begin{tabular}{|c|c|}
\hline Characteristic & Value \\
\hline \multicolumn{2}{|l|}{ Location, $n(\%)$} \\
\hline Head and neck & $68(45.3)$ \\
\hline Lower extremity and buttock & $36(24.0)$ \\
\hline Upper extremity & $30(20.0)$ \\
\hline Trunk & $7(4.7)$ \\
\hline Hand & $7(4.7)$ \\
\hline Foot & $2(1.3)$ \\
\hline \multicolumn{2}{|l|}{ Clinical size, $\mathrm{cm}, n(\%)$} \\
\hline$<1$ & $57(38.0)$ \\
\hline $1-2$ & $48(32.0)$ \\
\hline$>2$ & $43(28.7)$ \\
\hline Not specified & $2(1.3)$ \\
\hline \multicolumn{2}{|l|}{ Tumor thickness, $\mathrm{mm}(n=130)$} \\
\hline Mean (range) & $6.4(0.3-25)$ \\
\hline Median & 5 \\
\hline \multicolumn{2}{|c|}{ Anatomic level of invasion $(n=132), n(\%)$} \\
\hline II & $1(0.8)$ \\
\hline III & $3(2.3)$ \\
\hline IV & $56(42.4)$ \\
\hline V & $72(54.5)$ \\
\hline \multicolumn{2}{|l|}{ Mitotic rate per $\mathrm{mm}^{2}(n=103)$} \\
\hline Mean (range) & $29(1-96)$ \\
\hline \multicolumn{2}{|c|}{$\begin{array}{l}\text { Greatest horizontal histologic dimension, } \mathrm{mm} \\
(n=112)\end{array}$} \\
\hline Mean (range) & $9.5(0.8-45)$ \\
\hline \multicolumn{2}{|c|}{ Angiolymphatic invasion $(n=136), n(\%)$} \\
\hline Present & $45(33.1)$ \\
\hline Absent & $82(60.3)$ \\
\hline Equivocal & $9(6.6)$ \\
\hline \multicolumn{2}{|l|}{ Ulceration ( $n=123), n(\%)$} \\
\hline Present & $13(10.6)$ \\
\hline Absent & $107(87.0)$ \\
\hline Equivocal & $3(2.4)$ \\
\hline \multicolumn{2}{|l|}{ Growth pattern $(n=131), n(\%)$} \\
\hline Circumscribed & $70(53.4)$ \\
\hline Infiltrative & $61(46.6)$ \\
\hline
\end{tabular}

\section{Clinical Characteristics}

Many clinical characteristics were similar to those reported in other studies. The majority of patients diagnosed with MCC are older Caucasians. ${ }^{8-14}$ The mean age at diagnosis in our study was 70.3 . Our data suggested a minimal male to female predominance of 1.1:1, compared with that reported by Heath and colleagues of $1.4: 1 .^{10}$ In our study, $45.3 \%$ of lesions occurred on the head and neck and $50.0 \%$ on the extremities, including the buttocks. Others have reported frequencies of 29 to $62.5 \%$ on the head and neck and 33 to $52 \%$ on the extremities, including the buttocks. ${ }^{10,11,13}$

In our study, $9.5 \%$ of patients were immunosuppressed. Numerous studies support an association between MCC and immunosuppression. MedinaFranco and colleagues, in a review of seven studies, reported that $14.5 \%$ of patients had received or were receiving immunosuppressive therapy, and Heath and colleagues reported that $7.8 \%$ of their cohort were profoundly immunosuppressed (human immunodeficiency virus, chronic lymphocytic leukemia (CLL), solid organ transplant). ${ }^{10,13}$ Observations of MCC in transplant patients and patients with autoimmune disease taking immunosuppressant drugs indicate that long-term iatrogenic immunosuppression increases the risk of MCC. ${ }^{15}$ In the study by Heath and colleagues, age at diagnosis was comparable in immunosuppressed and immunocompetent patients, ${ }^{10}$ but in our study, immunosuppressed patients were on average more than 10 years younger than immunocompetent patients at diagnosis. In organ transplant patients, the mean age at diagnosis has been reported as $53 .^{16}$

Other malignancies have been identified with a high incidence in individuals with MCC. According to Howard and colleagues, in patients with other first primary cancers, the risk of developing MCC as a second primary malignancy was 1.36 times as great. ${ }^{17}$ In our study, $55.1 \%$ of patients had a diagnosis of a non-MCC cancer before diagnosis of MCC. History of a non-MCC skin cancer, including basal cell carcinoma (BCC), squamous cell carcinoma (SCC), and melanoma, occurred in $43.5 \%$ of patients. BCC occurred most commonly, followed by SCC and then melanoma. Others have also found a high incidence of skin cancers in patients with MCC. ${ }^{13,18,19}$ In our cohort, $21.1 \%$ of patients had a history of SCC, somewhat lower than the 34 to $41 \%$ in other reports that, unlike our study, included SCC after presentation of MCC. ${ }^{19-21}$ Twenty-eight $(19.0 \%)$ patients had a diagnosis of a non-skin cancer before diagnosis of MCC. Ten patients were diagnosed with a hematologic malignancy, 
lymphoma or CLL, both of which have been shown to carry a greater risk of MCC as a second primary malignancy. ${ }^{19,22}$

The majority of patients with MCC (70\%) present with disease clinically limited to the skin (stage I or II), 25\% with palpable regional lymphadenopathy (stage III), and 5\% with distant metastases (stage IV). ${ }^{7,11,13}$ The clinical staging of our patients at presentation was similar, with $54.5 \%$ with stage I disease, $19.0 \%$ with stage II, $20.4 \%$ with stage III, and $6.1 \%$ with stage IV. Clinical staging results in understaging in many patients with MCC. ${ }^{11}$ After pathologic staging with sentinel lymph node biopsy in more than $90 \%$ of our clinical stage I and II patients, the pathologic staging of our patients at presentation changed to $37.4 \%$ with stage I disease, $8.2 \%$ with stage II, $48.3 \%$ with stage III, and $6.1 \%$ with stage IV. Furthermore, if we included 14 patients with unknown primary tumors seen in the clinic during the study period, almost $60 \%$ of patients with MCC had regional or distant disease at presentation.

\section{Tumor Characteristics}

Tumor size is a dominant factor in staging, but until recently, use of multiple staging systems has led to confusion and inconsistencies among health care providers, patients, and researchers. ${ }^{23}$ In late 2009, the AJCC adopted a consensus staging system in which the maximum dimension of the tumor plays an important role in staging, ${ }^{7}$ but the method of measuring is not defined and may be interpreted as clinical (clinical size) or histologic (greatest histologic horizontal dimension). In this study, three distinct size measurements were recorded for each primary MCC — clinical size, greatest histologic horizontal dimension, and tumor thicknessalthough it remains to be determined whether one of these measurements is superior to the others at predicting outcome. Historically, clinically measured sizes have been the standard, yet as in our study, we would expect considerable variability in how clinical size is obtained. Ideally, study physicians would measure clinical size, but in referral medical centers, a partial or complete biopsy has often prompted patients' referral. In these instances, when available, we used measurements that referring physicians obtained. In other instances, we had only patient description or biopsy scar length available, which is a suboptimal means of measurement. In the literature, how clinical size measurement is obtained is frequently lacking but would be expected to be variable as well. This may have implications for the prognostic strength of clinical size in comparison with the other measurements of tumor size. Greatest histologic horizontal dimension would be expected to underestimate clinical size in part because of shrinkage that occurs with standard formalin-fixed permanent section tissue processing. ${ }^{24}$ In this study, the mean greatest histologic horizontal dimension was $9.07 \mathrm{~mm}$ for tumors with clinical size of 1 to $2 \mathrm{~cm}$ and $16.38 \mathrm{~mm}$ for tumors with clinical size $>2 \mathrm{~cm}$. In this study, if greatest histologic horizontal dimension rather than clinical size was used for staging, 18 primary MCCs would have been understaged. Tumor thickness has the potential to be a strong prognostic indicator. Recent studies report a positive association between greater tumor thickness and poor outcome. ${ }^{3}$ Other studies have found no correlation between tumor thickness and disease-free or overall survival. ${ }^{25}$ Using measurements from biopsy and re-excision if residual tumor is present, tumor thickness may be the most reproducible and reviewable. A consistent and systematic way to measure and report lesion size is critical for staging and downstream clinical decision-making and management.

In our series, tumors were smaller than those reported in the literature, with $38.0 \%$ of primary lesions smaller than $1 \mathrm{~cm}, 32.0 \% 1$ to $2 \mathrm{~cm}$, and $28.7 \%$ larger than $2 \mathrm{~cm}$ in clinical diameter. In the study by Heath and colleagues of patients diagnosed with MCC between 1980 and 2007, 21.3\% of primary lesions were smaller than $1 \mathrm{~cm}, 43.3 \%$ were 1 to $2 \mathrm{~cm}$, and $35.3 \%$ were larger than $2 \mathrm{~cm}$ in clinical diameter. ${ }^{10}$ Similarly, in our study, mean greatest histologic horizontal diameter was $9.5 \mathrm{~mm}$, compared with a mean of $20.1 \mathrm{~mm}$ reported in a 
TABLE 4. Clinical and Histologic Variables for Prospective Documentation in Primary Merkel Cell Carcinoma

\begin{tabular}{ll} 
Clinical Variables & Histologic Variables \\
\hline Sex & Greatest histologic horizontal dimension \\
& (in a transversely serially sectioned specimen) \\
Age & Tumor thickness (Breslow depth) \\
Race & Anatomic level of invasion (Clark level) \\
Immunosuppression status & Mitoses per $\mathrm{mm}^{2}$ (number of dermal mitoses in one $\mathrm{mm}^{2}$ ) \\
History of other cancers & Tumor growth pattern (circumscribed or infiltrative) \\
Clinical and pathologic stage at presentation & Ulceration \\
Tumor site & Angiolymphatic invasion \\
Tumor clinical size (greatest diameter) & \\
\hline
\end{tabular}

study of 156 patients over 25 years. ${ }^{3}$ It is likely that, with increased awareness of MCC and skin cancer in general, diagnosis occurs earlier in the disease course.

Various other histologic factors included in our primary MCC profile have been considered in analyses of prognostic variables in the literature, mostly small studies, including anatomic level of invasion, mitotic rate, growth pattern, ulceration, and angiolymphatic invasion..$^{3-5,26-30}$ In $96.9 \%$ of our patients, the primary MCC extended to anatomic level IV or V, and in $54.5 \%$, the tumor involved the subcutis (level V). In a study by Andea and colleagues, the deepest anatomic compartment involved by tumor was significantly associated with survival. ${ }^{3}$ Some smaller studies support this, but others do not. ${ }^{27,29,30}$

Few studies report on mitotic rate in primary MCC. In this study, the mean mitotic rate was 29/ $\mathrm{mm}^{2}$ (range $1-96 / \mathrm{mm}^{2}$ ). Skelton and colleagues found that higher mitotic rates were associated with lower survival rates, but two smaller studies failed to show a correlation. ${ }^{28-30}$ Our prior study found that greater mitotic rate was significantly associated with greater likelihood of a positive sentinel lymph node in MCC. ${ }^{31}$

In our cohort, $53.4 \%$ of tumors had a circumscribed growth pattern, and $46.6 \%$ had an infiltrative pattern. Histologic growth pattern has been shown to have prognostic significance in some studies. ${ }^{3,29}$ Andea and colleagues reported that a circumscribed pattern was associated with longer survival, whereas an infiltrative pattern had a poorer prognosis. ${ }^{3}$ We previously reported that an infiltrative pattern was significantly associated with a greater likelihood of a positive sentinel lymph node. ${ }^{31}$ Several small studies have not shown significance of histologic growth pattern as a prognostic marker in MCC. ${ }^{28,30}$

Ulceration was present in only $10.6 \%$ of primary MCCs in this cohort. Similarly, Andea and colleagues reported ulceration in $8 \%$ of their patients. ${ }^{3}$ Ulceration has not been shown to correlate significantly with prognosis., ${ }^{3,28-30}$

Angiolymphatic invasion has been reported to occur in 30 to $60 \%$ of MCCs and, in our study, occurred in $33.1 \% .^{3-5,28-30}$ In the study by Fields and colleagues, lymphovascular invasion was present in $56 \%$ of the primary tumors in which the status was reported. ${ }^{4}$ The discrepancy in the percentage of tumors with lymphovascular invasion between the study by Fields and colleagues and our study may be attributable to the use of immunohistochemistry in the former study to evaluate for angiolymphatic invasion in tumors initially found to be negative on hematoxylin and eosin evaluation. Andea and colleagues found lymphovascular invasion to be an independent predictor of survival on multivariate analysis, and in the study by Fields and colleagues, the presence of lymphovascular invasion was significantly associated with greater disease-specific death. ${ }^{3,4}$ Other studies have failed to show a significant correlation between angiolymphatic invasion and survival. ${ }^{28-30}$ 


\section{Conclusion}

This study presents a cross-sectional analysis of a modern prospectively maintained database with consistent pathology review. Histopathologic profiling of primary MCC in the literature is variable and limited, yet the importance of an accurate histopathologic profile for primary MCC cannot be overstated. ${ }^{6,32}$ Prospective documentation of these histologic parameters and clinical features, presented in Table 4, is needed to meaningfully analyze these for prognostic significance and to identify the important independent clinical and histologic features that best predict outcome. In the future, because of the rarity of the tumor, systematic prospective collection of detailed MCC data can be used to provide the framework for rigorous outcome studies and the ability to compare and share data from multiple sources.

\section{References}

1. The Rockville Merkel Cell Carcinoma Group. Merkel cell carcinoma: recent progress and current priorities on etiology, pathogenesis, and clinical management. J Clin Oncol 2009;27:4021-4026.

2. Hodgson NC. Merkel cell carcinoma: changing incidence trends. J Surg Oncol 2005;89:1-4.

3. Andea AA, Coit DG, Amin B, Busam KJ. Merkel cell carcinoma: histologic features and prognosis. Cancer 2008;113:2549-2558.

4. Fields RC, Busam KJ, Chou JF, Panageas KS, et al. Five hundred patients with Merkel cell carcinoma evaluated at a single institution. Ann Surg 2011;254:465-473.

5. Fields RC, Busam KJ, Chou JF, Panageas KS, et al. Recurrence and survival in patients undergoing sentinel lymph node biopsy for Merkel cell carcinoma: analysis of 153 patients from a single institution. Ann Surg Oncol 2011;18:2529-2537.

6. Bichakjian CK, Lowe L, Lao CD, Sandler HM, et al. Merkel cell carcinoma: critical review with guidelines for multidisciplinary management. Cancer 2007;110:1-12.

7. Merkel cell carcinoma. In: Edge SB, Byrd DR, Compton CC, Fritz AG, Greene FL, Trotti A, editors. AJCC cancer staging manual. New York, NY: Springer, 2009. pp. 315-323.

8. Albores-Saavedra J, Batich K, Chable-Montero F, Sagy N, et al. Merkel cell carcinoma demographics, morphology, and survival based on 3870 cases: a population based study. J Cutan Pathol 2010;37:20-27.

9. Stokes JB, Graw KS, Dengel LT, Swenson BR, et al. Patients with Merkel cell carcinoma tumors $<$ or $=1.0 \mathrm{~cm}$ in diameter are unlikely to harbor regional lymph node metastasis. J Clin Oncol 2009;27:3772-3777.
10. Heath M, Jaimes N, Lemos B, Mostaghimi A, et al. Clinical characteristics of Merkel cell carcinoma at diagnosis in 195 patients: the AEIOU features. J Am Acad Dermatol 2008;58:375-381.

11. Allen PJ, Bowne WB, Jaques DP, Brennan MF, et al. Merkel cell carcinoma: prognosis and treatment of patients from a single institution. J Clin Oncol 2005;23:2300-2309.

12. Agelli M, Clegg LX. Epidemiology of primary Merkel cell carcinoma in the United States. J Am Acad Dermatol 2003;49:832-841.

13. Medina-Franco H, Urist MM, Fiveash J, Heslin MJ, et al. Multimodality treatment of Merkel cell carcinoma: case series and literature review of 1024 cases. Ann Surg Oncol 2001;8:204-208.

14. Akhtar S, Oza KK, Wright J. Merkel cell carcinoma: report of 10 cases and review of the literature. J Am Acad Dermatol 2000;43:755-767.

15. Agelli M, Clegg LX, Becker JC, Rollison DE. The etiology and epidemiology of Merkel cell carcinoma. Curr Probl Cancer 2010;34:14-37.

16. Penn I, First MR. Merkel's cell carcinoma in organ recipients: report of 41 cases. Transplantation 1999;68:1717-1721.

17. Howard RA, Dores GM, Curtis RE, Anderson WF, et al. Merkel cell carcinoma and multiple primary cancers. Cancer Epidemiol Biomarkers Prev 2006;15:1545-1549.

18. Kaae J, Hansen AV, Biggar RJ, et al. Merkel cell carcinoma: incidence, mortality, and risk of other cancers. J Natl Cancer Inst 2010;102:793-801.

19. Brenner B, Sulkes A, Rakowsky E, Feinmesser M, et al. Second neoplasms in patients with Merkel cell carcinoma. Cancer 2001;91:1358-1362.

20. Walsh NM. Primary neuroendocrine (merkel cell) carcinoma of the skin: morphologic diversity and implications thereof. Hum Pathol 2001;32:680-689.

21. Gomez LG, DiMaio S, Silva EG, Mackay B. Association between neuroendocrine (merkel cell) carcinoma and squamous cell carcinoma of the skin. Am J Surg Pathol 1983;7:171-177.

22. Vlad R, Woodlock TJ. Merkel cell carcinoma after chronic lymphocytic leukemia: case report and literature review. Am J Clin Oncol 2003;26:531-534.

23. Sarnaik AA, Lien MH, Nghiem P, Bichakjian CK. Clinical recognition, diagnosis, and staging of merkel cell carcinoma, and the role of the multidisciplinary management team. Curr Probl Cancer 2010;34:38-46.

24. Dauendorffer JN, Bastuji-Garin S, Guero S, et al. Shrinkage of skin excision specimens: formalin fixation is not the culprit. $\mathrm{Br} \mathrm{J}$ Dermatol 2009;160:810-814.

25. Goldberg SR, Neifeld JP, Frable WJ. Prognostic value of tumor thickness in patients with Merkel cell carcinoma. J Surg Oncol 2007;95:618-622.

26. Andea A, Coit DG, Busam K. An analysis of morphologic parameters as prognostic markers in Merkel cell carcinoma. Am J Dermatopathol 2006;28:228. 
27. Sandel HD 4th, Day T, Richardson MS, Scarlett M, et al. Merkel cell carcinoma: does tumor size or depth of invasion correlate with recurrence, metastasis, or patient survival? Laryngoscope 2006;116:791-795.

28. Llombart B, Monteagudo C, Lopez-Guerrero JA, Carda C, et al. Clinicopathological and immunohistochemical analysis of 20 cases of Merkel cell carcinoma in search of prognostic markers. Histopathology 2005;46:622-634.

29. Mott RT, Smoller BR, Morgan MB. Merkel cell carcinoma: a clinicopathologic study with prognostic implications. J Cutan Pathol 2004;31:217-223.

30. Skelton HG, Smith KJ, Hitchcock CL, McCarthy WF, et al. Merkel cell carcinoma: analysis of clinical, histologic, and immunohistologic features of 132 cases with relation to survival. J Am Acad Dermatol 1997;37:734-739.
31. Schwartz JL, Griffith KA, Lowe L, Wong SL, et al. Features predicting sentinel lymph node positivity in Merkel cell carcinoma. J Clin Oncol 2011;29:1036-1041.

32. Brown JA, Smoller BR. Merkel cell carcinoma: what is it, what will it do and where will it go? what role should the pathologist play in reporting this information? J Cutan Pathol 2009;36:924-927.

Address correspondence and reprint requests to: Jennifer L. Schwartz, MD, University of Michigan Health System, 1910 Taubman Center, 1500 E. Medical Center Dr., Ann Arbor, MI 48109, or e-mail: jennschw@med.umich.edu 